\title{
The tumor suppressor NOR1 suppresses cell growth, invasiveness, and tumorigenicity in glioma
}

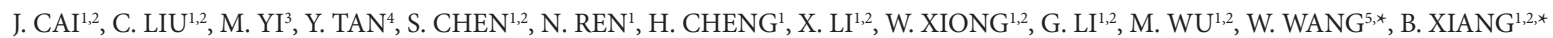

${ }^{1}$ Hunan Provincial Cancer Hospital and Cancer Hospital Affiliated to Xiangya Medical School, Central South University, Changsha, Hunan, China; ${ }^{2}$ Cancer Research Institute, Xiangya School of Medicine, Central South University, Changsha, Hunan, China; ${ }^{3}$ Department of Dermatology, Xiangya Hospital, Central South University, Changsha, Hunan, China; ${ }^{4}$ Department of Dermatology, Second Xiangya Hospital, The Central South University, Hunan Key Laboratory of Medical Epigenetics, Changsha, Hunan, China; ${ }^{5}$ Department of Pathology, Affiliated Hospital of Jining Medical University, Jining, Shandong, China

*Correspondence: xiangbolin@csu.edu.cn; wangwei1985@csu.edu.cn

Received July 24, 2019 / Accepted November 13, 2019

\begin{abstract}
Oxidored-nitro domain-containing protein 1 (NOR1) is a tumor suppressor downregulated in various human cancers, including nasopharyngeal carcinoma (NPC), lung cancer, and testicular cancer. NOR1 protein is highly expressed in the normal brain; however, its role in brain tumors remains unknown. In this study, we demonstrated that the NOR1 protein level was decreased in glioma tissue samples as compared to its normal counterpart. Exogenously expressed NOR1 protein in glioma U251 cells inhibits tumor cell proliferation, migration, and invasion. Re-expression of NOR1 induced cell cycle $\mathrm{S}$ to G2 phase arrest and suppressed its tumorigenicity in nude mice. Overexpression of NOR1 in U251 cells also led to a decrease of Ki67 expression in xenografts. Transcriptomic analysis revealed that NOR1 expression altered the expression of genes favored cell proliferation. Among the differentially expressed genes, FOXR2, a member of the FOX gene family, which promotes glioma progression, was decreased in NOR1 expressing cells. The downregulation of FOXR2 by NOR1 was validated in vitro and in vivo. Our findings suggest for the first time that NOR1 suppresses glioma progression via modulating the FOXR2 expression.
\end{abstract}

Key words: glioma, FOXR2, NOR1, tumor suppressor

Glioma is one of the most common types of primary malignancy in the central nervous system seriously threatening human health $[1,2]$. Malignant glioma, a highly invasive tumor, causes an annual incidence of 3-5 per 100,000 individuals, usually in adults over 45 years of age $[3,4]$. The main clinical symptom is presented as memory loss, headaches, seizures, focal neurological deficits, and even personality disruptions. Glioma can diffusely invade and infiltrate surrounding brain tissue, and intertwine each other among native cells [5]. Due to its invasive and therapy-resistant nature, most of these tumors are incurable. Generally, gliomas fall into three categories: oligodendrogliomas, astrocytomas, and ependymomas [6]. Approximately half of all newly diagnosed glioma is considered as glioblastoma, which belongs to astrocytomas and is the most malignant type of brain cancer. Despite the advance of multimodal therapeutics, the overall five-year survival rate of glioma patients, especially those with high-grade glioblastoma multiforme, is not optimistic; most of those patients only have 1-year median survival rate [7].
The oxidored-nitro domain-containing protein 1(NOR1, also called organic solute carrier partner 1, or OSCP1) is a putative tumor suppressor gene, which was downregulated in several kinds of cancers, such as nasopharynx, gastric, colon, rectum and cervical cancers. The main epigenetic reason for NOR1 silencing in these cancers may be attributed to the hypermethylation of NOR1 promoter, which has been observed in NPC and hematological malignancies [8]. NOR1 gene is located in a $120 \mathrm{~kb}$ region at $1 \mathrm{p} 34.3$ and was isolated from NPC for the first time in 2003 [9]. The protein encoded by this downregulated gene possesses a great deal of structural similarity to the nitroreductase of Salmonella typhimurium. NOR1 protein upregulated mitochondrial $\mathrm{Bax} / \mathrm{Bcl}-2$ ratio, thus enabling cytochrome $\mathrm{C}$ release to the cytoplasm and triggering apoptosis induced by hypoxia [10]. When overexpressed in Drosophila eye imaginal discs, NOR1 induced endoplasmic reticulum stress and apoptosis thus leading to a rough eye phenotype in adult flies [11]. Re-expression of NOR1 suppresses NPC cell growth and tumorigenicity in nude mice through inhibiting autophagy 
and glycolysis [12]. In addition, to suppress cancer cell proliferation, re-expression of NOR1 in NPC cells resulted in the reversal of epithelial-mesenchymal transition and suppressed tumor invasiveness in vitro and metastasis in vivo [13-15]. Human NOR1 protein, a solute carrier protein that mediates various kinds of organic solutes in a $\mathrm{pH}$-dependent and sodium-independent manner, is highly expressed in the normal brain, especially in neurons [16]. So far, the function of NOR1 in epithelial cells derived cancers has been intensively studied. However, its role in tumor development of the central nervous system is still remaining elusive.

In this study, we demonstrated NOR1 protein was decreased in glioma. Low expression of NOR1 protein predicted unfavorable clinical outcome. Overexpression of NOR1 in glioma suppressed U251 cells proliferation, migration, and invasiveness in vitro, and their tumorigenicity in nude mice.

\section{Patients and methods}

Tumor tissue samples from patients. A cohort of 236 subjects with glioma, as well as non-cancerous brain tissue control subjects, were recruited between July 2008 and May 2017 from the Pathology Department of the Affiliated Hospital of Jining Medical University (Shandong, China), and their tissue samples were used for immunohistochemical analysis of NOR1 expression. Clinicopathological data were also collected from patients' medical records, including gender, age, clinical stage, lymph node metastasis, and pathology diagnosis. The age of all patients ranged from 4 to 81 years (mean age of 50.56 years). 119 participants (50.42\%) were males and 117 (49.58\%) were females. In accordance with the WHO classification system, 6 cases were WHO I, 84 cases were WHO II, 45 cases were WHO III and 74 cases were WHO IV. This study was approved by the Institute Research Ethics Committee for the use of clinical samples and each patient signed a consent form to participate in the study.

Immunohistochemistry. NOR1 protein was measured by immunohistochemical staining as described previously [17, 18]. Briefly, immunohistochemistry assay was performed using the peroxidase-antiperoxidase technique after a microwave antigen retrieval procedure. Sections were incubated with polyclonal anti-NOR1 (\#12598-1-AP, Proteintech Group, Chicago, USA), anti-Ki67 (\#E607238, BBI Life Sciences, Shanghai, China) and anti-FOXR2 (\#14111-1-AP, Proteintech Group, Chicago, USA) antibodies overnight at $4^{\circ} \mathrm{C}$. A staining index (values, $0-6$ ) obtained as the staining intensity (scores: negative $=0$, weak $=1$, moderate $=2$, or strong $=3$ ) and $\%$ of staining of tumor cells (scores: $<10 \%=1$, $10-50 \%=2,>50 \%=3$ ) were calculated. The sum of these two scores was used as the final immunoreactive score (0-6), i.e., low expression ( $0-1$ scores) and high expression (2-6 scores).

Cell culture and lentivirus infection. U251, a worldwide broadly used glioma cell line, was routinely maintained in Dulbecco's modified Eagle's medium (DMEM) supplemented with $10 \%$ fetal bovine serum (FBS), $100 \mathrm{U} / \mathrm{ml}$ penicillin G, and $100 \mu \mathrm{g} / \mathrm{ml}$ streptomycin (GIBCO, Grand Island, NY) in a humidified incubator at $37^{\circ} \mathrm{C}$ with $5 \% \mathrm{CO}_{2}$ and $95 \%$ air. NOR1 was stably introduced into U251 cell line by using a NOR1-expressing lentivirus. Stable transfectants were selected by puromycin for 1 week. A GFP expressing lentivirus was used as control.

RNA isolation, reverse transcription and quantitative real-time PCR. Total RNA from cells was extracted using Trizol (Invitrogen, San Diego, CA, USA) according to the manufacturer's protocol. The residual DNA was removed by treating with DNase I (Roche Diagnostics, Rotkreuz, Switzerland). Total RNA ( $3 \mu \mathrm{g})$ was reverse-transcribed to cDNA with M-MLV Reverse Transcriptase Kit (Thermo Fisher, Rockford, IL, USA). The resulted cDNA was used for real-time PCR with SYBR Green qPCR Master Mix (Thermo Fisher) on CFX96 Touch ${ }^{\text {tx }}$ Real-Time PCR Detection System (BioRad) following the manufacturer's instruction. The thermal cycling conditions included an initial denaturation step at $95^{\circ} \mathrm{C}$ for $30 \mathrm{~s}$ and 40 cycles of $95^{\circ} \mathrm{C}$ for $5 \mathrm{~s}$ followed by $60^{\circ} \mathrm{C}$ for $30 \mathrm{~s}$. The results of three independent experiments were used to calculate the average value. The relative NOR1 mRNA levels were calculated by normalization to GAPDH mRNA levels. The PCR primers were as follows: NOR1 forward, 5'-TCAAGGGATTCATCCGAGAC-3'; NOR1 reverse, 5'-CTGGCCAAGAAATTCAGCTC-3'; FOXR2 forward, 5'-TCCCTCCAGTCCCCTGAAAT-3'; FOXR2 reverse, 5'-GCTAGGGCAATAAGGTGGCT-3'; GAPDH forward, 5'-AACGGATTTGGTCGTATTGG-3'; GAPDH reverse, 5'-TTGATTTTGGAGGGATCTCG-3'.

Protein extraction and western blot. Total cellular protein was extracted from the cultured cells using a lysis buffer (Beyotime, Jiangsu, China) and equal amount of protein samples were separated in sodium dodecyl sulfate polyacrylamide gel electrophoresis (SDS-PAGE) and transferred onto a PVDF membrane (Millipore, Billerica, MA). The membranes were immunoblotted with the following antibodies: polyclonal anti-NOR1 (Proteintech Group, Chicago, IL, USA), anti-FOXR2 (Proteintech Group, Inc., Chicago, IL) and anti-GAPDH antibodies (Proteintech Group, Inc., Chicago, IL).

Cell viability assay. A proliferation assay was carried out using Cell Counting Kit-8 (CCK-8, Dojindo Laboratories, Japan) according to the manufacturer's protocol. U251 cells infected with NOR1 expressing lentivirus or scrambled lentivirus were seeded into 96-well plates at a density of $2 \times 10^{3}$ cells per well and grown for $0,24,48,72 \mathrm{~h}$. $10 \mu \mathrm{l}$ CCK-8 reagent was added to each well and the cells were incubated at $37^{\circ} \mathrm{C}$ for $2 \mathrm{~h}$. The absorbance was recorded at $450 \mathrm{~nm}$ with a microplate reader (Bio-Rad, Hercules, CA). GraphPad Prism 5 was used for drawing the proliferation curves.

Colony formation assay. Approximately 2,000 cells were seeded into six-well plates in triplicate and incubated for 10-14 days. Colonies were stained with crystal violet and counted. 
Wound healing assay. Cell suspensions were seeded onto a 6 -well plate at a density of $8 \times 10^{5}-1 \times 10^{6}$ cells per well. Monolayer cell cultures were scratched by using a $10 \mu \mathrm{l}$ tip, and then the non-adhered cells were removed by washing with PBS. Cells were allowed for growth for $24 \mathrm{~h}$ in serumfree medium. Cell migration was observed by using a microscope.

Migration and invasion assays. Tumor cells migration and invasion assays were performed as described previously [18]. Briefly, cells were seeded onto transwell membrane inserts ( $8 \mu \mathrm{m}$, Corning) in a serum-free medium. 15\% FBS containing media was added to the lower chamber. After $6 \mathrm{~h}$ to $24 \mathrm{~h}$ incubation at $37^{\circ} \mathrm{C}$, cells that migrated to the lower surface of the membrane were fixed in $4 \%$ paraformaldehyde and stained with $0.5 \%$ crystal violet. For each membrane, 5 random fields were counted at $\times 10$ magnification. The mean was calculated and data are presented as mean \pm SEM from three independent experiments performed in triplicate. For the tumor cell invasion assay, the membrane was pre-coated with $15 \mu$ lMatrigel (BDBiosciences, Bedford, MA), and the rest of the method was identical to the tumor cell migration assay except incubation of cells was conducted at 24 to $48 \mathrm{~h}$ at $37^{\circ} \mathrm{C}$.

Cell cycle assay. U251 cells were washed with cold phosphate-buffered saline (PBS) and then fixed with 75\% ethanol overnight at $-20^{\circ} \mathrm{C}$. The fixed cells were washed with cold PBS and then stained with propidium iodide (PI, Beyotime, Shanghai, China) for $30 \mathrm{~min}$ at $37^{\circ} \mathrm{C}$ in the dark. The stained cells were assessed using a FACS Calibur flow cytometer (BD Bioscience, San Jose, CA).

RNA-Seq. Total RNAs were extracted using TRIzol reagent (Invitrogen). The integrity of the RNA was monitored using Bioanalyzer 2100. RNA-Seq was performed using BGISEQ500 sequencer. A two-fold cutoff was used to identify differentially expressed genes between two cell populations. Differentially expressed genes between the two groups were identified by the NOISeq method. GO terms enrichment was analyzed using DAVID (https://david.ncifcrf.gov/) and Gene Set Enrichment Analyses (GSEA) [19, 20].

Xenograft tumor formation assay. An animal experiment protocol was approved by the ethics review committee of the Central South University of China. For nude mouse tumor cell xenograft model, tumor cells were harvested using trypsin-EDTA, washed with DMEM, resuspended in serumfree DMEM, and the suspended cells $\left(1 \times 10^{6} / 0.2 \mathrm{ml}\right)$ were subcutaneously injected into 4 to 5 -week old male BALB/c nude mice $(\mathrm{n}=4$; Shanghai SLAC Laboratory Animal Co. Ltd., Shanghai, China). Tumor growth was monitored by measuring the tumor sizes with a caliper. The tumor volume $(\mathrm{V})$ was calculated according to the formula: $\mathrm{V}=0.5 \times$ (length $\times$ width $^{2}$ ). Nude mice were sacrificed at 12 weeks after tumor cell inoculation or when the tumor became moribund. The xenograft tumors were fixed in $4 \%$ saline-buffered formalin, embedded in paraffin, sectioned at $4 \mu \mathrm{m}$, and then stained with $\mathrm{H} \& \mathrm{E}$. A minimum of 15 sections was examined per mouse under a light microscope.
Statistical analysis. Differences between groups were estimated by using Pearson's $\mathrm{c} 2$ to analyze the association of NOR1 expression with clinicopathological characteristics by using the SPSS 18.0 software package (SPSS, Chicago, IL). The Kaplan-Meier method was used to determine the probability of survival and data was analyzed with the logrank test by using GraphPad Prism software (version 6, La Jolla, CA). A p-value $<0.05$ was considered significant.

\section{Results}

A decrease of NOR1 protein in glioma samples predicts unfavorable clinical outcome. In this study, NOR1 protein level was detected in all glioma samples and surrounding normal brain tissue samples adjacent to glioma. Strong cytoplasmic staining for NOR1 protein was detected in 45 out of $48(93.8 \%)$ normal brain tissue samples (Figure 1A). A substantial portion of glioma samples (194 of 236; 82.2\%, $\mathrm{p}<0.001$ ) showed weak NOR1 staining (Figure 1B), but only a minority (42 of $236 ; 17.8 \%$ ) of glioma samples displayed strong NOR1 staining (Figure 1C). This data indicated that NOR1 protein was downregulated in glioma compared to normal brain tissue. We then analyzed the relationship between NOR1 expression and clinicopathological data of glioma patients. NOR1 expression is associated with an early clinical stage in glioma patients (Table S1). Kaplan-Meier analysis revealed that low NOR1 expression was associated with poorer overall survival compared to patients with high NOR1 level in 118 glioma patients $(\mathrm{p}=0.0413$ respectively, Figure 1D). There was a $40 \%$ reduction in overall survival of patients with a low level of NOR1 expression compared to those patients with a high level of NOR1 expression. Thus, our data suggested that the downregulation of NOR1 contributed to the development and progression of glioma.

Stable expression of NOR1 inhibits U251 cells proliferation in vitro. To study the effect of NOR1 on glioma cells, the human NOR1 gene was stably introduced into U251 cells with a NOR1 expressing lentivirus. The NOR1 expression level was determined by qRT-PCR and western blot (Figures $2 \mathrm{~A}, 2 \mathrm{~B})$. Growth curve assays indicated that exogenous NOR1 dramatically inhibited U251 cells growth (Figure 2C). Colony formation assay revealed that the re-expression of NOR1 significantly inhibited tumor cell colony formation ( $<<0.001$, Figures 2D, 2E). We also analyzed the effect of NOR1 expression on cell cycle progression. Flow cytometry assay revealed that overexpression of NOR1 induced cell cycle $S$ to G2 phase arrest in U251 cells (Figure 2F). Thus, our data suggested that NOR1 acted as a tumor suppressor in glioma.

Re-expression of NOR1 suppressed migration and invasiveness progression of U251 cells. To get further insights into the biological relevance of NOR1 expression, we analyzed its effect on the migrative and invasive behavior of U251 cells. The wound-healing assay revealed that NOR1-expressing U251 cells exhibited significantly reduced mobility compared to vector control cells (Supplementary 
A

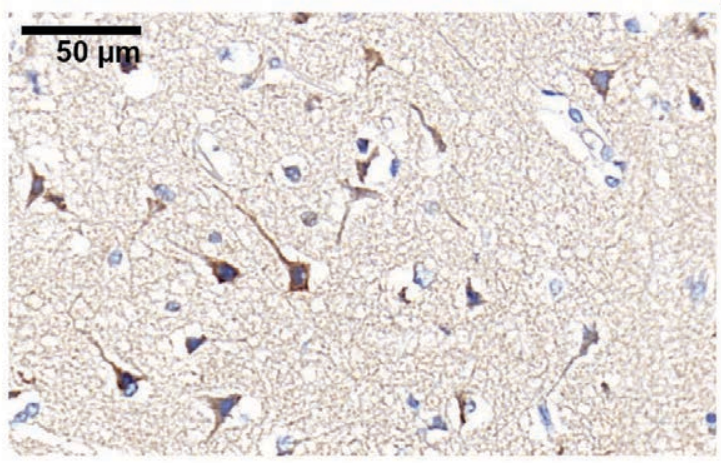

B

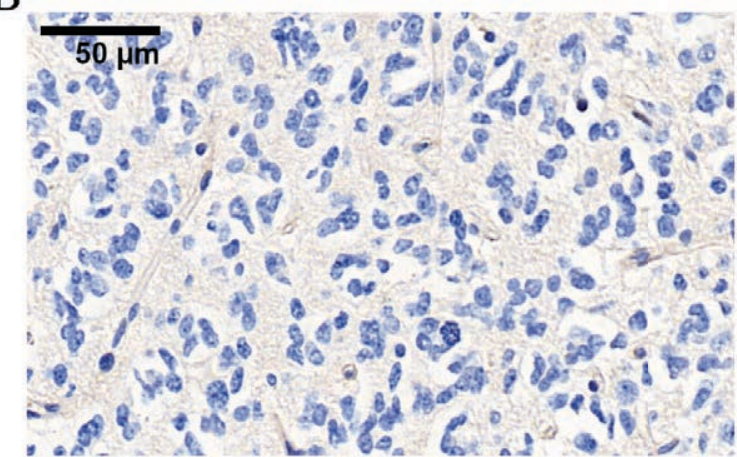

$\mathrm{D}$

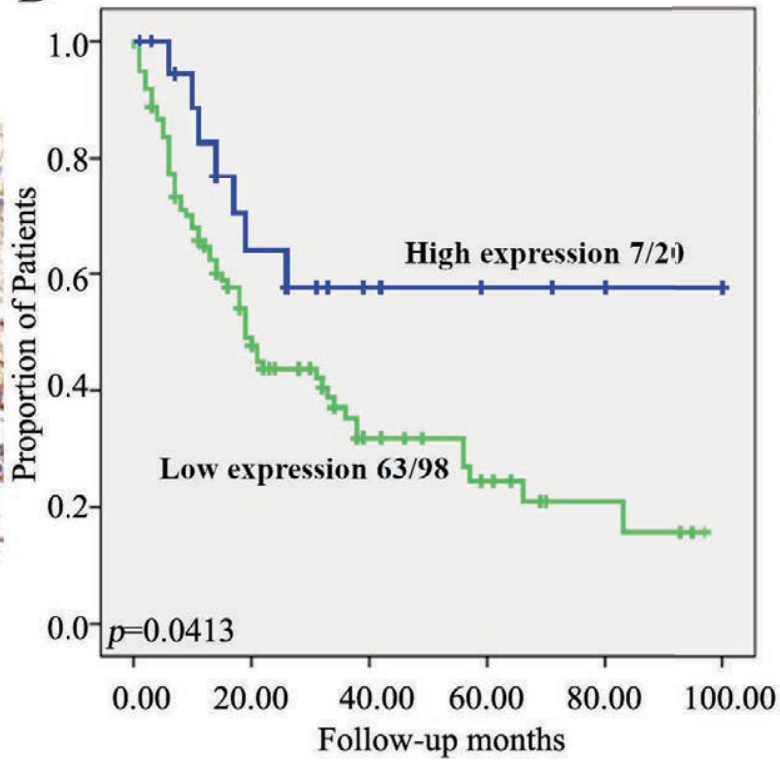

Figure 1. The loss of NOR1 protein in glioma predicts an unfavorable prognosis. A) Strong immunostaining of NOR1 protein in the normal brain. B) Weak staining of NOR1 in glioma. C) Strong staining of NOR1 in glioma. D) Overall survival of NPC patients stratified according to NOR1 protein expression. A staining color obtained as the expression intensity for NOR1 protein (color: negative $=$ colorless, weak $=$ yellow, moderate $=$ brown, or strong = tawny).

Figure S1). Migration assays clearly indicated that NOR1 expression suppressed U251 cell migration (Figure 3A). We also performed the Matrigel-coated Boyden chamber invasion assay. The results demonstrated that NOR1 expression decreased glioma cell invasiveness (Figure 3B). qPCR assay showed that NOR1 expression suppressed the expression of mesenchymal markers (Vimentin, Twist, Fibronectin, Figure 3C). Thus, our data suggested that NOR1 suppressed migration and invasiveness in glioma.

Stable expression of NOR1 in U251 cells suppresses their tumorigenicity in vivo. We then asked whether NOR1 suppressed glioma malignant behavior in vivo. Human tumor xenograft models revealed that NOR1 expression suppressed the tumorigenicity of U251 cells in nude mice (Figures 4A, 4B). Xenografts from NOR1-expressing cells growled slowly and were much smaller than that from the control cells (Figures 4C, 4D). Hematoxylin and eosin staining suggested that NOR1 promoted glioma cell differentiation with a decreased nucleus-to-cytoplasm ratio, well-organized tumor architecture in glioma cells (Figure 4E). Immunohistochemical staining demonstrated fewer Ki67 cells in tumors from NOR1/U251 cells (Figures 4F, 4G). These data indicated that NOR1 suppressed tumorigenicity U251 cell in vivo.

NOR1 overexpression inhibits FOXR2 expression. To address the downstream effects of NOR1 expression, we performed RNA-Seq analysis on NOR1/U251 and NC/U251 cells. Using three biological replicates, we found 77 differentially regulated genes (including 34 upregulated and 43 downregulated genes) with a false discovery rate of $p<0.05$ upon NOR1 expression in U251 cells (Figure 5A). KEGG analysis revealed these differentially expressed genes correlated with metabolism (Figure 5B). GSEA analysis showed the gene expression pattern in NOR1-expressing U251 cells was correlated with the epithelial to mesenchymal transition 
A

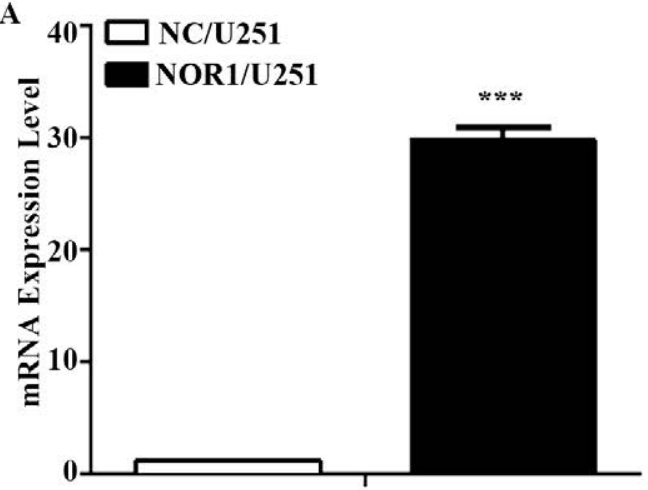

C

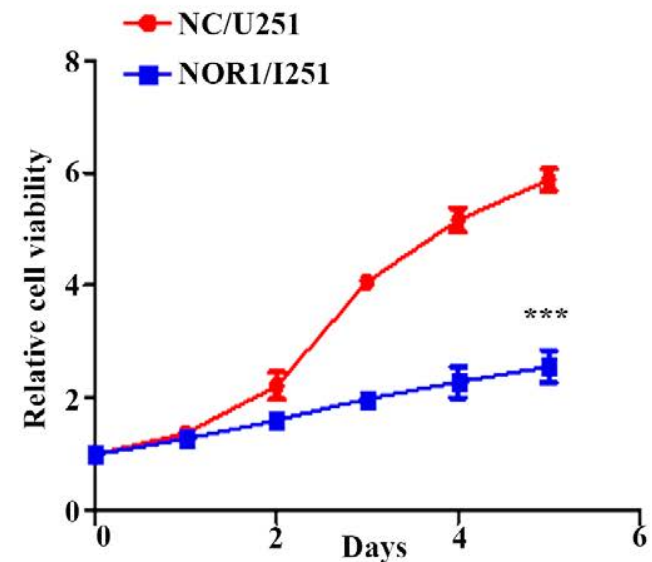

$\mathbf{F}$

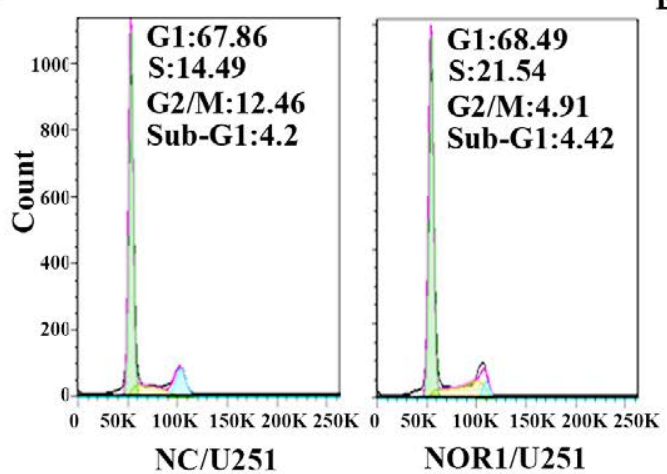

B

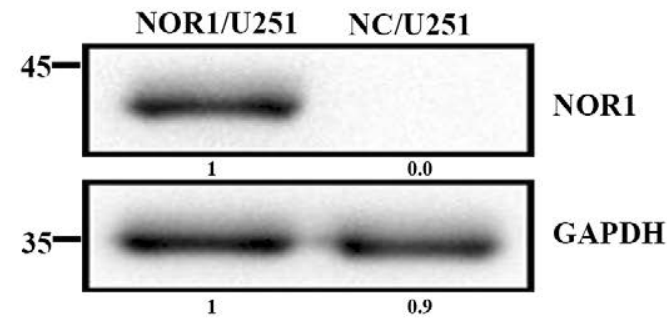

D

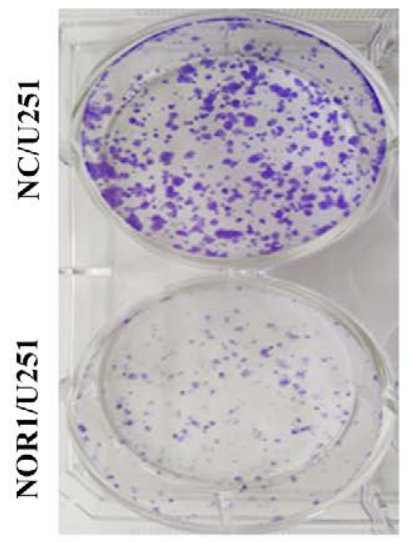

E

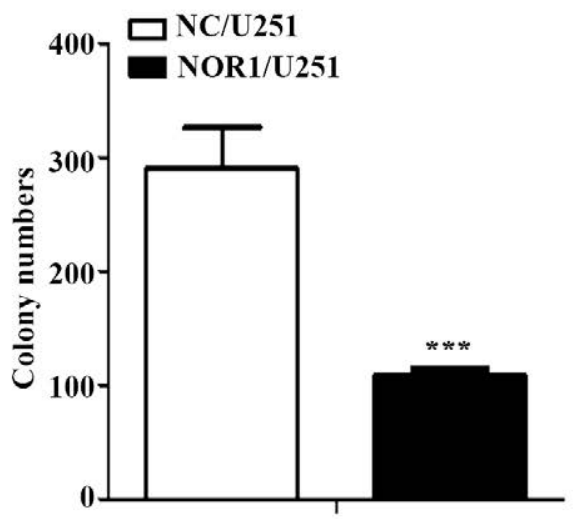

Figure 2. The re-expression of NOR1 protein suppresses glioma cell proliferation and cell cycle in vitro. A) NOR1 mRNA levels in U251 cells stably transfected with a NOR1-lentivirus were determined by GPCR. B, the NOR1 protein levels in U251 cells were measured by western blotting. C) CCK8 assay showed that NOR1 overexpressing inhibited cell growth. D, E) Colony-forming ability of U251 cells with stable-express of NOR1. F) Cell cycle analysis showed S/G2 arrest in U251 cells after re-expressing of NOR1. ${ }^{* * *} \mathrm{p}<0.001$ compared to the control cell.

progression signaling pathway (Figure 5C). We evaluated the RNA-Seq data by RT-qPCR (Figure 5D). As shown in Figure 5D, FOXR2, LASP1, PDGFA, and RUNX3 were dramatically reduced in NOR1-expressing U251 cells. We further demonstrated FOXR2 protein was dramatically reduced in NOR1-expressing U251 cells (Figure 5E). Immunohistochemical staining demonstrated increased FOXR2 protein in tumors from NOR1/U251 cells (Figure 5F). This result suggested that NOR1 suppressed glioma progression via modulating FOXR2 expression.

\section{Discussion}

In this study, we demonstrated that the tumor suppressor NOR1 is frequently decreased in glioma. Loss of NOR1 predicted unfavorable clinical outcomes. NOR1 suppressed 
A
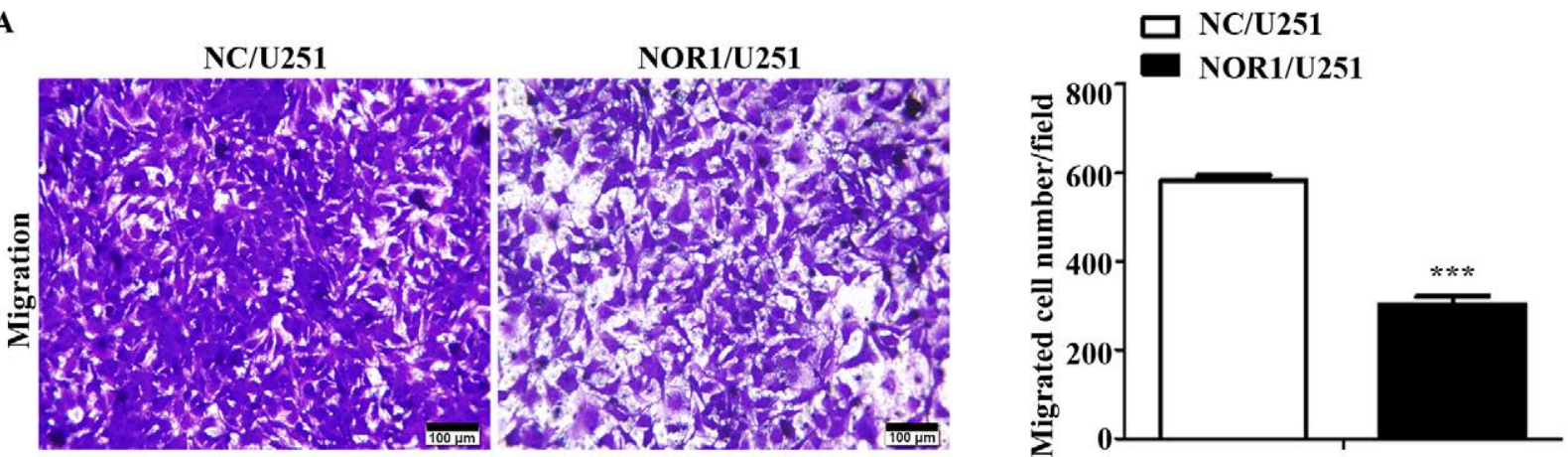

B
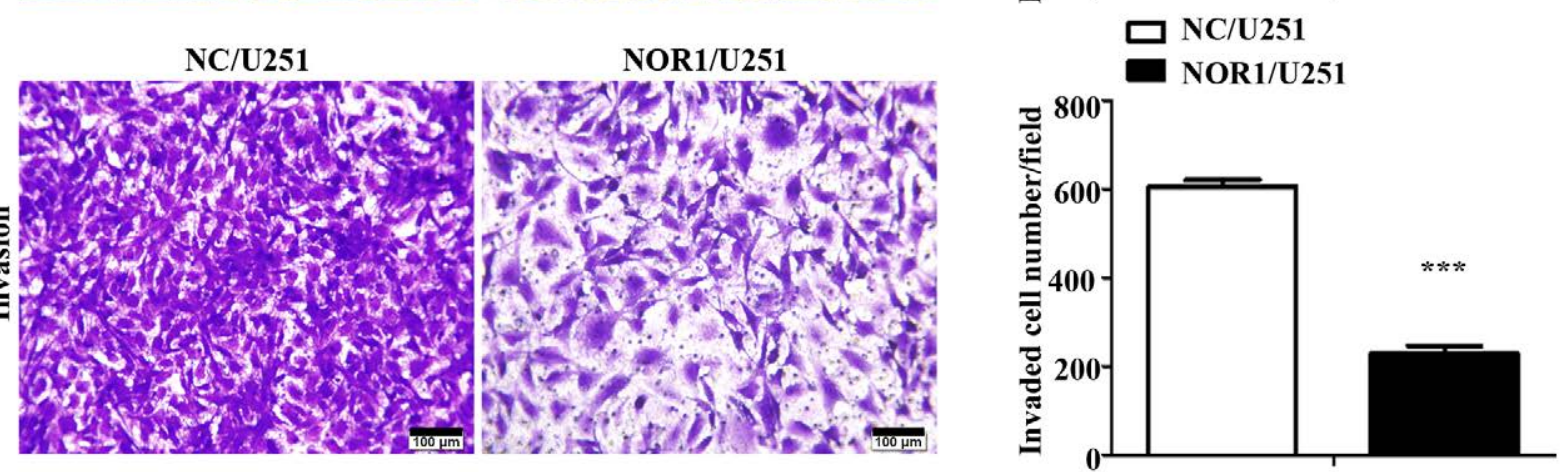

C

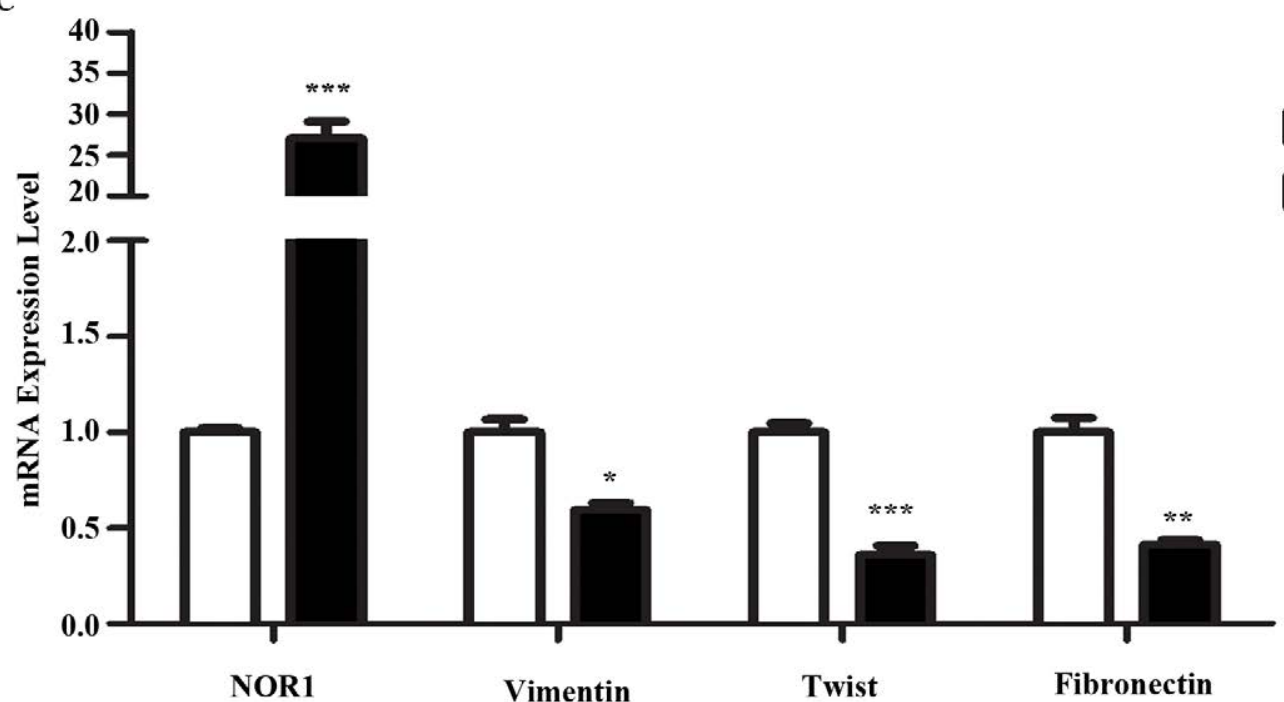

Figure 3. The re-expression of NOR1 protein suppresses glioma cell migration and invasiveness. A) Migration assay showed re-expressing NOR1 inhibits U251 cell migration. B) Invasion assay showed re-expressing NOR1 inhibits U251 cell invasiveness. C) Real-time RT-PCR assay showed NOR1 differentially regulates mesenchymal relative gene expression in $\mathrm{U} 251$ cells. ${ }^{*} \mathrm{p}<0.05,{ }^{* *} \mathrm{p}<0.01,{ }^{* * *} \mathrm{p}<0.001$ compared to the control cell.

glioma cell proliferation and invasiveness in vitro. NOR1 suppressed tumorigenicity in vivo. We further depicted that FOXR2, an oncogene, was downregulated by NOR1 in glioma.

NOR1, a novel gene containing a nitroreductase domain, was the first nasopharyngeal epithelial-specific tumor suppressor gene [21]. Early studies found that the coding region single nucleotide polymorphism in the coding region of the NOR1 gene is associated with the pathogenesis of
NPC, suggesting that the NOR1 gene is the NPC susceptibility gene [22]. Restoring NOR1 expression in HNE1 cells has the effect of inhibiting tumor cell growth and proliferation. Hypermethylation of the NOR1 gene promoter is one of the reasons for its downregulation in NPC tissues and cells. Oxidative stress can initiate NOR1 transcription by activating the transcription factors HSF1 (heat shock factor 1) and NRF1 (nuclear respiratory factor 1), inhibiting autophagy to promote apoptosis and preventing tumorigenesis [9]. 
A

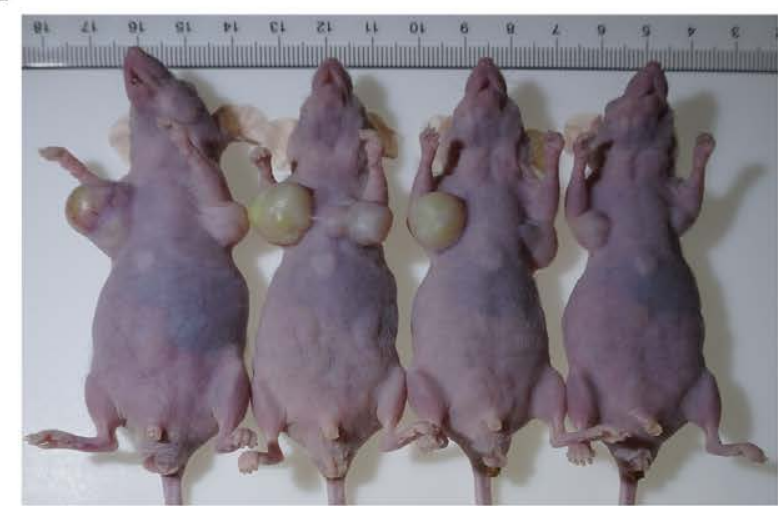

B

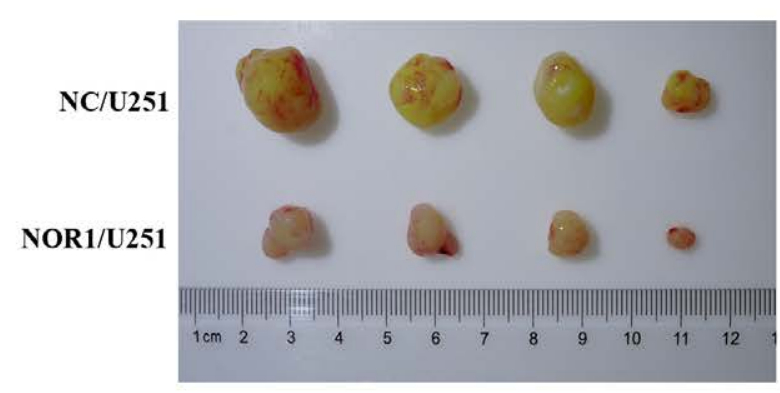

C

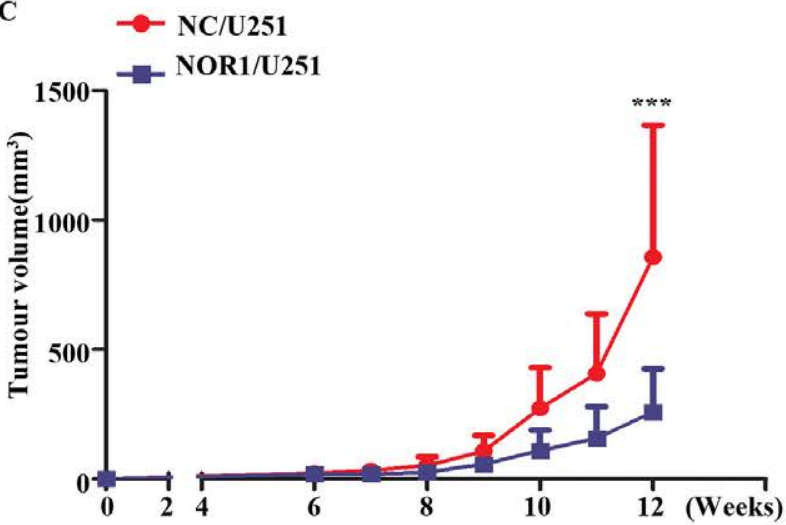

D

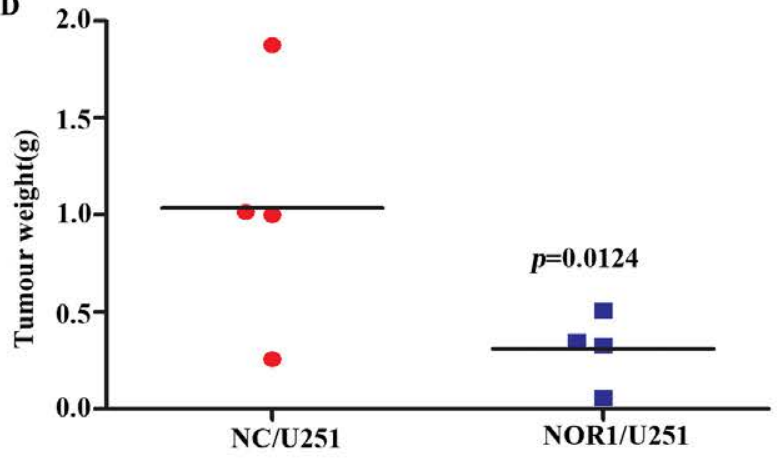

$\mathbf{E}$
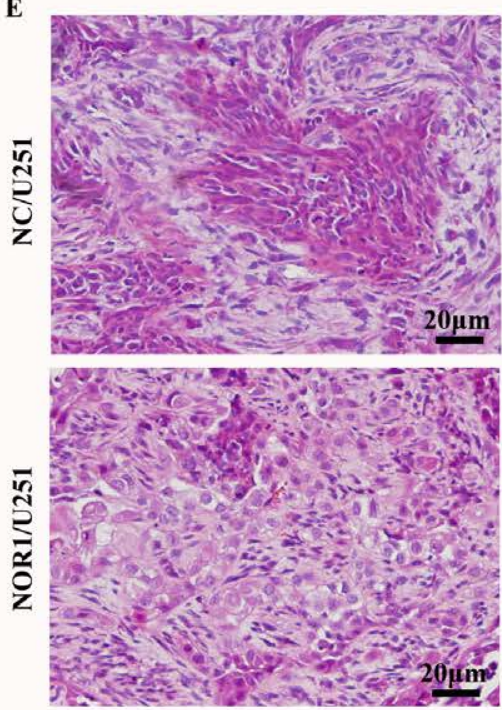

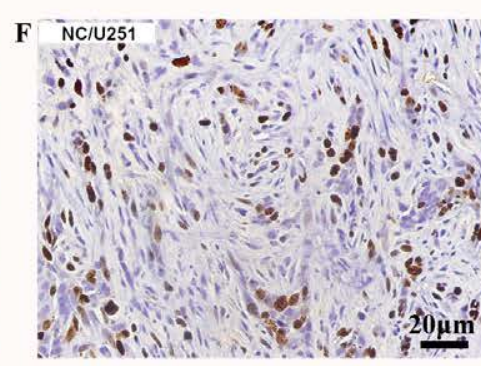

$G$

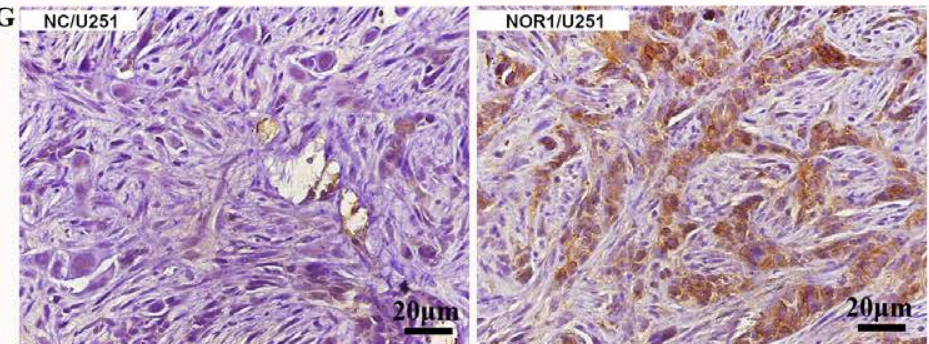

Figure 4. A stable re-expression of NOR1 suppresses tumorigenicity of glioma cells in nude mice. A) Inhibition of growth of tumor xenografts in nude mice by stable expressing of NOR1 in U251 cells. B) Comparison of tumor weight between the control and NOR1-expressing groups. C) Growth curve showing the effect of NOR1-expressing on xenograft growth in nude mice. D) Tumor weight showing the effect of NOR1-expressing on xenograft growth in nude mice. E, H) Staining of xenograft tissues from the control and NOR1-expressing groups. F, G) Immunohistochemical staining showed Ki67 and NOR1 expression in vivo. A staining color obtained as the expression intensity for Ki67 and NOR1 protein (color: negative = colorless, weak $=$ yellow, moderate $=$ brown, or strong $=$ tawny). ${ }^{* * *} \mathrm{p}<0.001$ compared to the control cell.

NOR1 inhibits tumor cell Slug transcription by FOXA1 and HDAC2, up-regulates the expression of epithelial keratin cytokeratin 4 and cytokeratin 13, promotes cytoskeletal remodeling and inhibits epithelial-to-mesenchymal transi- tion [23]. The above studies indicate that the NOR1 gene is a candidate for a new tumor suppressor gene.

NOR1 is conserved in the evolution of multiple species, studies have confirmed that NOR1 is highly expressed in 


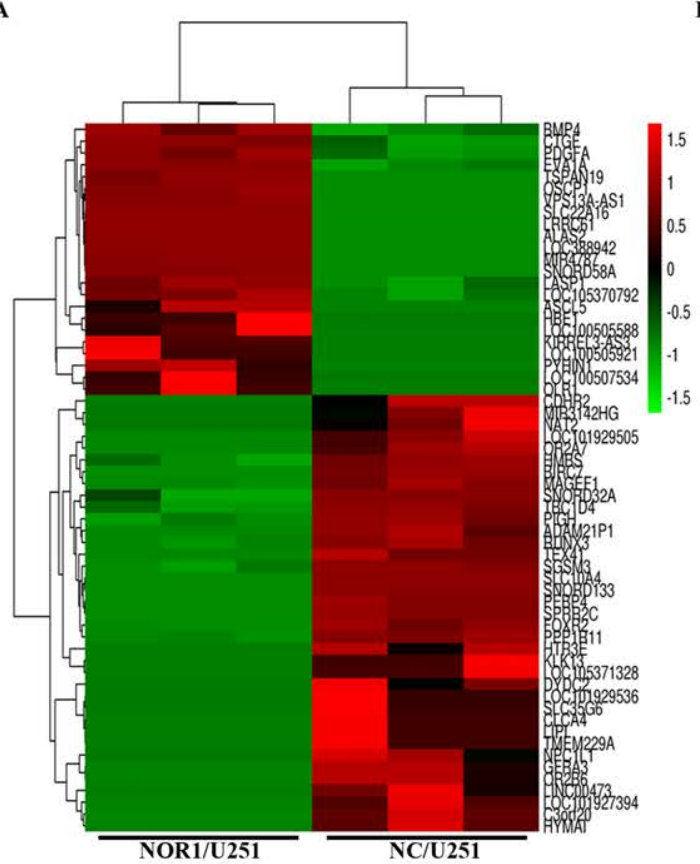

D

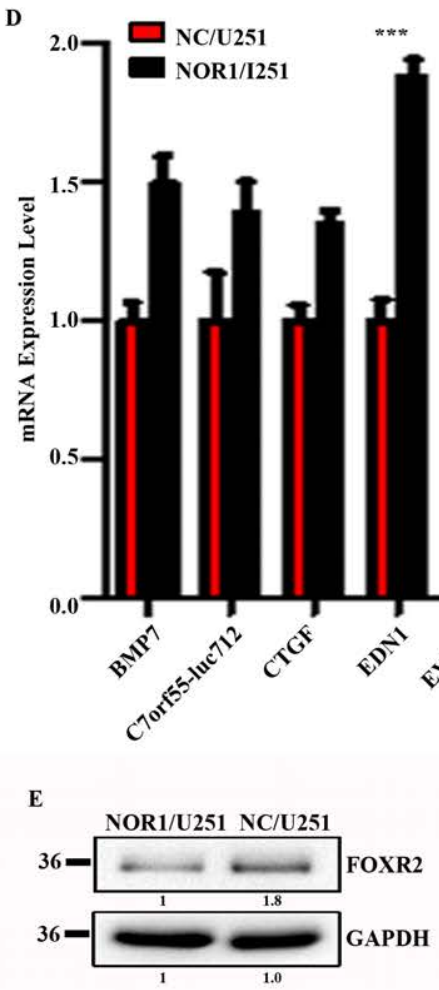

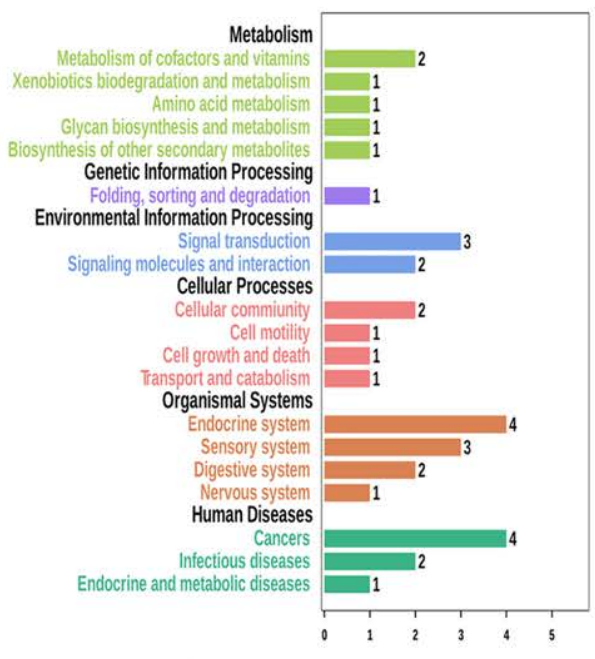

C

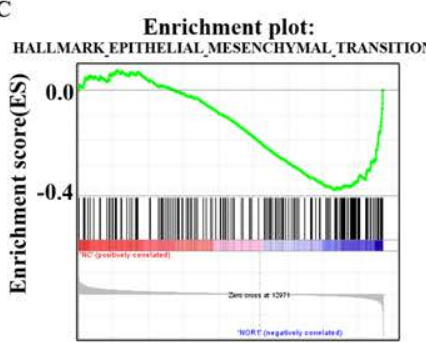

Figure 5. The re-expression of NOR1 protein suppresses glioma FOXR2 expression. A) Heatmap showed expression data for representative DEGs between NOR1-expressing or NC-U251 cells. B) KEGG analysis different expression genes. C) GSEA analysis showed RNA-Seq data from NOR1/U251 and NC/U251 cells. D) Real-time RT-PCR assay showed NOR1 differentially regulates FOXR2 expression in U251 cells. E) Western blot showed NOR1 differentially regulates FOXR2 expression in U251 cells. F) Immunohistochemical staining showed that FOXR2 expression in vivo. A staining color obtained as the expression intensity for FOXR2 protein (color: negative $=$ colorless, weak $=$ yellow, moderate $=$ brown, or strong $=$ tawny). ${ }^{\star} \mathrm{p}<0.05$, ${ }^{* *} \mathrm{p}<0.01,{ }^{* * *} \mathrm{p}<0.001$ compared to the control cell. 
human brain, testis, nasopharynx, bronchial mucosa, and is hardly expressed in tissues such as heart, liver, spleen, kidney, stomach, and skeletal muscle [16]. NOR1 is highly expressed in normal brain, but low in glioma, suggesting that NOR1 plays an important role in the development of glioma.

NOR1 protein was expressed in all the brain regions at different levels, especially higher levels in neurons, suggesting a role of NOR1 in brain development. We also depicted that NOR1 was decreased in most glioma samples. Furthermore, loss of NOR1 is associated with metastasis and predicted the poor prognosis of glioma patients. Our data are similar to the observation that the loss of NOR1 expression independently predicted an unfavorable overall survival in NPC.

Human Forehead-box (FOX) gene family consists of at least 43 members [24]. Forkhead-box (FOX) superfamily genes are implicated in carcinogenesis through gene amplification, retroviral integration and chromosomal translocation $[25,26]$. Forkhead box R2 (FOXR2, also named as FOXN6), a member of the FOX gene family, is associated with a variety of biological processes such as embryogenesis, differentiation, proliferation, transformation, apoptosis, tumorigenesis and so on [27]. As the transcription factor human FOXR2, FOXN6 has been identified and characterized by human genome sequence RP11-167p23 (AL159987) by bioinformatics methods [28]. By combining the results of a large-scale proteomic analysis of the human transcription factor interaction network with knowledge databases, studies identified FOXR2 as one of the top-ranked candidate proto-oncogenes. FOXR2 is highly expressed in breast cancer samples and is associated with poor prognosis. The role of FOXR2 as an oncogene in medulloblastoma, colorectal cancer [29], endometrial adenocarcinoma [30], prostate cancer [31], breast cancer [28], hepatocellular carcinoma cell [32]. In addition, FOXR2 was identified as an oncogene in malignant peripheral nerve sheath tumors and medulloblastoma and promoted the proliferation of granule neuron precursor cells [33].

In conclusion, we depicted that NOR1 is highly expressed in the brain. Loss of NOR1 protein is common in glioma. NOR1 acts as a tumor suppressor in glioma. NOR1 inhibits the proliferation and clonality of glioma cells by interacting with FOXR2, causing $S$ to G2 arrest, inhibiting tumor migration and invasion, and reducing cell tumorigenicity. Our study provides new insight for NOR1 and a new target for the comprehensive treatment of glioma.

Supplementary information is available in the online version of the paper.

Acknowledgments: This study was supported in part by grants from The National Natural Science Foundation of China (81572667, $81772902,81872278,81703131,81802945)$, the National "111" Project (Project \#111-2-12), The Natural Science Foundation of Hunan Province, China (2018JJ1040, 2017JJ3440, 2019JJ40182), The research program of Health Commission of Hunan Province (20201067, 20201040), and the Hunan Provincial Key Research and Development Program (2018SK2130, 2018SK2131).

\section{References}

[1] CAPDEVILA C, RODRIGUEZ VL, MARTI J. Glioblastoma Multiforme and Adult Neurogenesis in the Ventricular-Subventricular Zone: A Review. J Cell Physiol 2017; 232: 15961601. https://doi.org/10.1002/jcp.25502

[2] CHAUDHURI S, SINGH MK, BHATTACHARYA D, DATTA A, HAZRA I et al. T11TS immunotherapy repairs PI3KAKT signaling in T-cells: Clues toward enhanced T-cell survival in rat glioma model. J Cell Physiol 2018; 233: 759-770. https://doi.org/10.1002/jcp.26047

[3] SIMONELLI M, PERSICO P, PERRINO M, ZUCALI PA, NAVARRIA $P$ et al. Checkpoint inhibitors as treatment for malignant gliomas: "A long way to the top". Cancer Treat Rev 2018; 69: 121-131. https://doi.org/10.1016/j.ctrv.2018.06.016

[4] WATKINS S, SONTHEIMER H. Unique biology of gliomas: challenges and opportunities. Trends Neurosci 2012; 35: 546-556. https://doi.org/10.1016/j.tins.2012.05.001

[5] CHENG L, WU Q, GURYANOVA OA, HUANG Z, HUANG $\mathrm{Q}$ et al. Elevated invasive potential of glioblastoma stem cells. Biochem Biophys Res Commun 2011; 406: 643-648. https:// doi.org/10.1016/j.bbrc.2011.02.123

[6] LAPOINTE S, PERRY A, BUTOWSKI NA. Primary brain tumours in adults. Lancet 2018; 392: 432-446. https://doi. org/10.1016/S0140-6736(18)30990-5

[7] LINZ U. Effects of radiotherapy with concomitant and adjuvant temozolomide versus radiotherapy alone on survival in glioblastoma in a randomised phase III study: 5-year analysis of the EORTC-NCIC trial. Cancer 2010; 116: 1844-1846. https://doi.org/10.1002/cncr.24950.

[8] YI M, YANG J, LI W, LI X, XIONG W et al. The NOR1/ OSCP1 proteins in cancer: from epigenetic silencing to functional characterization of a novel tumor suppressor. J Cancer 2017; 8: 626-635. https://doi.org/10.7150/jca.17579

[9] LI W, LI X, WANG W, LI X, TAN Y et al. NOR1 is an HSF1- and NRF1-regulated putative tumor suppressor inactivated by promoter hypermethylation in nasopharyngeal carcinoma. Carcinogenesis 2011; 32: 1305-1314. https://doi. org/10.1093/carcin/bgr174

[10] XIANG B, YI M, LI W, WANG W, ZHENG P et al. Expression of oxidored nitro domain-containing protein 1(NOR1) impairs nasopharyngeal carcinoma cells adaptation to hypoxia and inhibits PDK1 expression. Mol Cell Biochem 2014; 393: 293-300. https://doi.org/10.1007/s11010-014-2072-9

[11] HUU NT, YOSHIDA H, YAMAGUCHI M. Overexpression of tumor suppressor protein OSCP1/NOR1 induces ER stress and apoptosis during development of Drosophila melanogaster. Am J Cancer Res 2015; 5: 1718-1729.

[12] LI W, LI X, WANG W, YI M, ZHOU Y et al. Tumor suppressor gene Oxidored-nitro domain-containing protein 1 regulates nasopharyngeal cancer cell autophagy, metabolism, and apoptosis in vitro. Int J Biochem Cell Biol 2013; 45: 2016-2026. https://doi.org/10.1016/j.biocel.2013.06.020

[13] WANG W, LI X, ZHANG W, LI W, YI M et al. Oxidorednitro domain containing protein 1 (NOR1) expression suppresses slug/vimentin but not snail in nasopharyngeal carcinoma: Inhibition of EMT in vitro and in vivo in mice. Cancer Lett 2014; 348: 109-118. https://doi.org/10.1016/j. canlet.2014.03.005 
[14] CHEN S, YOUHONG T, TAN Y, HE Y, BAN Y et al. EGFR-PKM2 signaling promotes the metastatic potential of nasopharyngeal carcinoma through induction of FOSL1 and ANTXR2. Carcinogenesis 2019; bgz180. https://doi. org/10.1093/carcin/bgz180

[15] WANG W, YI M, CHEN S, LI J, ZHANG H et al. NOR1 Suppresses Cancer Stem-Like Cells Properties of Tumor Cells via the Inhibition of the AKT-GSK-3beta-Wnt/betacatenin-ALDH1A1 Signal Circuit. J Cell Physiol 2017; 232: 2829-2840. https://doi.org/10.1002/jcp.25706

[16] XIANG B, WANG W, LI W, LI X, LI X et al. Differential expression of oxidored nitro domain containing protein 1 (NOR1), in mouse tissues and in normal and cancerous human tissues. Gene 2012; 493: 18-26. https://doi. org/10.1016/j.gene.2011.11.039

[17] CHEN S, ZHENG P, WANG W, YI M, CHEN P et al. Abberent expression of NOR1 protein in tumor associated macrophages contributes to the development of DEN-induced hepatocellular carcinoma. J Cell Physiol 2018; 233: 50025013. https://doi.org/10.1002/jcp.26349

[18] CAI J, CHEN S, YI M, TAN Y, PENG Q et al. $\triangle \mathrm{Np63} \alpha$ is a super enhancer-enriched master factor controlling the basalto-luminal differentiation transcriptional program and gene regulatory networks in nasopharyngeal carcinoma. Carcinogenesis 2019; bgz203. https://doi.org/10.1093/carcin/bgz203

[19] HUANG DW, SHERMAN BT, LEMPICKI RA. Systematic and integrative analysis of large gene lists using DAVID bioinformatics resources. Nat Protoc 2009; 4: 44-57. https://doi. org/10.1038/nprot.2008.211

[20] DEBRABANT B. The null hypothesis of GSEA, and a novel statistical model for competitive gene set analysis. Bioinformatics 2017; 33: 1271-1277. https://doi.org/10.1093/bioinformatics/btw803

[21] NIE X, ZHANG B, LI X, XIANG J, XIAO B et al. Cloning, expression, and mutation analysis of NOR1, a novel human gene down-regulated in HNE1 nasopharyngeal carcinoma cell line. J Cancer Res Clin Oncol 2003; 129: 410-414. https://doi.org/10.1007/s00432-003-0451-9

[22] HUANG H, ZHOU YF, JIANG PZ, SHEN XM, YAO KT. Screening of single nucleotide polymorphisms in nasopharyngeal carcinoma associated genes by denaturing high-performance liquid chromatography. Di Yi Jun Yi Da Xue Xue Bao 2002; 22: 602-604.
[23] WANG W, YI M, CHEN S, LI J, LI G et al. Significance of the NOR1-FOXA1/HDAC2-Slug regulatory network in epithelial-mesenchymal transition of tumor cells. Oncotarget 2016; 7: 16745-16759. https://doi.org/10.18632/oncotarget.7778

[24] KATOH M, KATOH M. Human FOX gene family (Review). Int J Oncol 2004; 25: 1495-1500.

[25] LI J, WANG W, CHEN S, CAI J, BAN Y et al. FOXA1 reprograms the TGF- $\beta$-stimulated transcriptional program from a metastasis promoter to a tumor suppressor in nasopharyngeal carcinoma. Cancer Lett 2019; 442: 1-14. https://doi. org/10.1016/j.canlet.2018.10.036

[26] PENG Q, ZHANG L, LI J, WANG W, CAI J et al. FOXA1 Suppresses the Growth, Migration, and Invasion of Nasopharyngeal Carcinoma Cells through Repressing miR-1005p and miR-125b-5p. J Cancer 2020; 11: 2485-2495. https:// doi.org/10.7150/jca.40709

[27] WANG X, HE B, GAO Y, LI Y. FOXR2 contributes to cell proliferation and malignancy in human hepatocellular carcinoma. Tumour Biol 2016; 37: 10459-10467. https://doi. org/10.1007/s13277-016-4923-3

[28] LI X, WANG W, XI Y, GAO M, TRAN M et al. FOXR2 Interacts with MYC to Promote Its Transcriptional Activities and Tumorigenesis. Cell Rep 2016; 16: 487-497. https://doi. org/10.1016/j.celrep.2016.06.004

[29] LU SQ, QIU Y, DAI WJ, ZHANG XY. FOXR2 Promotes the Proliferation, Invasion, and Epithelial-Mesenchymal Transition in Human Colorectal Cancer Cells. Oncol Res 2017; 25: 681-689. https://doi.org/10.3727/09650401 6X14771034190471

[30] DENG X, HOU C, LIANG Z, WANG H, ZHU L et al. miR202 Suppresses Cell Proliferation by Targeting FOXR2 in Endometrial Adenocarcinoma. Dis Markers 2017; 2017: 2827435. https://doi.org/10.1155/2017/2827435

[31] XU W, CHANG J, LIU G, DU X, LI X. Knockdown of FOXR2 suppresses the tumorigenesis, growth and metastasis of prostate cancer. Biomed Pharmacother 2017; 87: 471-475. https://doi.org/10.1016/j.biopha.2016.12.120

[32] WANG X, HE B, GAO Y, LI Y. FOXR2 contributes to cell proliferation and malignancy in human hepatocellular carcinoma. Tumour Biol 2016; 37: 10459-10467. https://doi. org/10.1007/s13277-016-4923-3

[33] KOSO H, TSUHAKO A, LYONS E, WARD JM, RUST AG et al. Identification of FoxR2 as an oncogene in medulloblastoma. Cancer Res 2014; 74: 2351-2361. https://doi. org/10.1158/0008-5472.CAN-13-1523 


\section{The tumor suppressor NOR1 suppresses cell growth, invasiveness, and tumorigenicity in glioma}

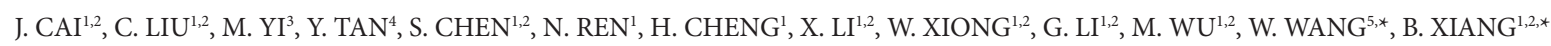

\section{Supplementary Information}

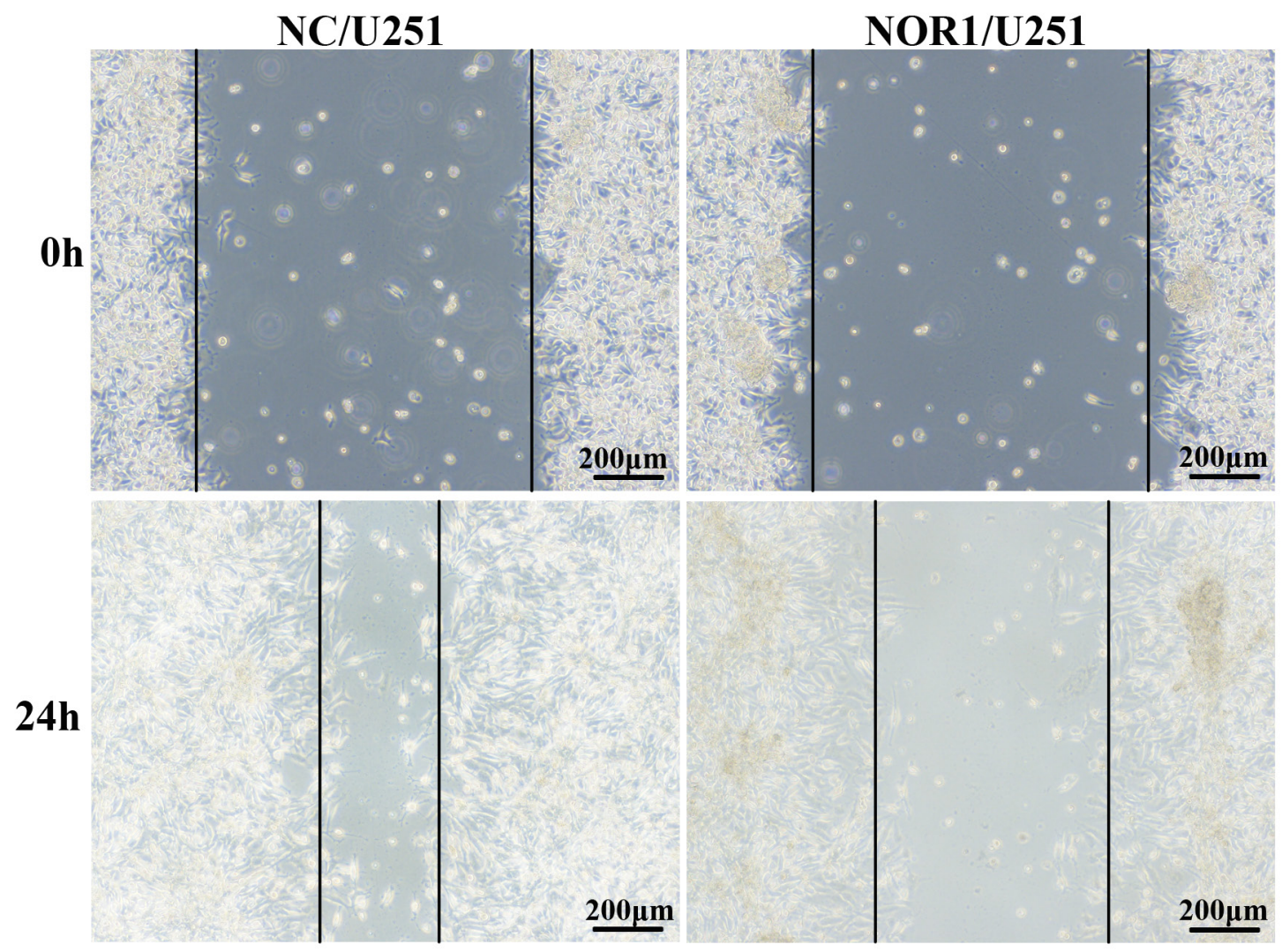

Supplementary Figure S1. The re-expression of NOR1 protein suppresses glioma cell migration. Wound healing assay showed the re-expression of NOR1 inhibits U251 cell migration.

\begin{tabular}{lccc}
$\begin{array}{l}\text { Supplementary Table S1. Relationship between NOR1 expression and } \\
\text { clinicopathological data of glioma patients. }\end{array}$ \\
\cline { 1 - 2 } Characteristics & \multicolumn{2}{c}{ Expression of NOR1 protein } & \multirow{2}{*}{ p-value } \\
\cline { 2 - 3 } & \multicolumn{1}{c}{ High } & Low & \\
\hline $\begin{array}{l}\text { Age (years) } \\
\leq 48\end{array}$ & $19(18.3 \%)$ & $85(81.75)$ & 0.866 \\
$>48$ & $23(17.45)$ & $109(82.6 \%)$ & \\
Gender & & & \\
$\quad$ Male & $22(18.5 \%)$ & $97(81.5 \%)$ & 0.780 \\
Female & $20(17.1 \%)$ & $97(82.69 \%)$ & \\
Clinical stage & & & \\
I+II & $26(24.5 \%)$ & $80(75.5 \%)$ & 0.0057 \\
III+IV & $10(9.7 \%)$ & $93(90.3 \%)$ & \\
\hline
\end{tabular}

